\title{
Rotation and the wind momentum-luminosity relation for extragalactic distances
}

\author{
A. Maeder \\ Geneva Observatory, 1290 Sauverny, Switzerland \\ Received 12 February 2001 / Accepted 27 April 2001

\begin{abstract}
The effects of axial stellar rotation on the wind-momentum relation (WLR) for determining the extragalactic distances are investigated. Despite the fact that the mass loss rates grow quite a lot with rotation, remarkably the effects on the WLR are found to be very small on average. As an example, for an average orientation angle between the rotation axis and the line of sight, the luminosity would be overestimated by $5.9 \%$ for a star rotating at $90 \%$ of its break-up rotational velocity. Different orientation angles between the rotation axis and the line of sight produce some limited scatter.
\end{abstract}

Key words. stars: distances - stars: mass loss - stars: variables: general - stars: winds and outflows - galaxies: distances and redshifts

\section{Introduction}

There is an important relation between the mechanical wind momentum $\dot{M} v_{\infty}$ of the mass outflow and the luminosity $L$ of hot stars predicted by the theory of radiative winds (e.g. Kudritzki et al. 1989; Kudritzki 2000; Kudritzki \& Puls 2000). This relation writes

$\dot{M} v_{\infty} \propto R^{-0.5} L^{\frac{1}{\alpha}}$,

where $\dot{M}$ is the mass loss rate, $v_{\infty}$ the terminal velocity and $R$ the stellar radius The parameter $\alpha$ is equal to about $\frac{2}{3}$, it is a so-called force-multiplier representing the power law exponent of the distribution of line strengths of the spectral lines driving the wind. The observations of O-type stars in the Galaxy and the LMC have shown the existence of a tight relationship between the wind momentum and the luminosity (Puls et al. 1996). More recently, the existence of a wind momentum-luminosity relation (WLR) has also been confirmed for A- and B-type supergiants (Kudritzki et al. 1999).

The potentialities of the WLR for the determination of extragalactic distances are claimed to be great (Kudritzki 2000). The WLR provides absolute luminosities from direct spectroscopic measurements of the mass loss rates and of the terminal velocities obtained from the line profiles. Kudritzki (2000) states that this method "may allow independent distance moduli to be obtained with an accuracy of $10 \%$ out to the Virgo and Fornax clusters of galaxies".

* e-mail: Andre.Maeder@obs.unige.ch
Rotation has been shown to increase significantly the mass loss rates of a star of a given mass and luminosity, i.e. up to a factor 2 and 4 for stars of 40 and $60 \mathrm{M}_{\odot}$, (Maeder 1999; Maeder \& Meynet 2000). For stars close to the Eddington-limit, rotation may even increase the mass loss rates by orders of magnitude with respect to the non rotating case. Also, rotation modifies the terminal velocities $v_{\infty}$ and the wind densities according to the colatitude $\vartheta$. In view of these large effects of rotation, we may wonder about the effects of rotation on the WLR. This may also be particularly useful if the proportion of fast rotators is higher in lower metallicity galaxies, as suggested by some recent observations (Maeder et al. 1999; Keller 2000).

\section{Rotational effects on the WLR}

\subsection{Classical case}

In the classical case without rotation, the mass loss rates and the terminal velocities behave as (Puls et al. 1996; Kudritzki 2000)

$$
\begin{aligned}
& \dot{M} \propto L^{\frac{1}{\alpha}}[M(1-\Gamma)]^{1-\frac{1}{\alpha}} \\
& v_{\infty} \propto 2.24 \frac{\alpha}{1-\alpha}\left[\frac{G M(1-\Gamma)}{R}\right]^{0.5}
\end{aligned}
$$

$\Gamma=\frac{\kappa_{\mathrm{es}} L}{4 \pi c G M}$ is the Eddington factor with $\kappa_{\mathrm{es}}$ the electronscattering opacity. The WLR is thus

$\dot{M} v_{\infty} \propto \frac{1}{R^{0.5}} L^{\frac{1}{\alpha}}[M(1-\Gamma)]^{\frac{3}{2}-\frac{1}{\alpha}}$. 
Thus, with the value $\alpha=2 / 3$ typical for O-stars, one obtains the WLR relation given by Eq. (1).

\subsection{Local WLR}

In the case of rotating stars, the stellar shape defined by the Roche model has to be accounted for. For a star of angular velocity $\Omega$, the surface equation is a function of a parameter $\omega^{2}=\frac{\Omega^{2} R_{\mathrm{eb}}^{3}}{G M}$, where $\Omega$ is the angular velocity and $\omega$ the fraction of the angular velocity at break-up. $R_{\mathrm{eb}}$ is the equatorial radius at break-up. The von Zeipel theorem is used for defining the local flux, with possibly some corrections for differential rotation (Maeder 1999). The Eddington factor $\Gamma_{\Omega}(\vartheta)$ in a rotating star must be defined as the ratio of the local flux to the local limiting flux (Maeder \& Meynet 2000). We recall that in the framework of the wind theory, the Eddington factor appearing in the various expressions is that for electron scattering opacity $\kappa_{\mathrm{es}}$, since the true opacities are expressed as a function of $\kappa_{\text {es }}$ by means of the force multipliers (Kudritzki \& Puls 2000). In this specific context, $\Gamma_{\Omega}$ is the same at all latitudes

$\Gamma_{\Omega}=\frac{\Gamma}{1-\frac{\Omega^{2}}{2 \pi G \rho_{\mathrm{m}}}}$,

where $\Gamma$ is the Eddington factor defined above and $\rho_{\mathrm{m}}$ is the average density inside the surface equipotential. We also stress that the critical velocity is not given by $v_{\text {crit }}=\left[\frac{G M}{R}(1-\Gamma)\right]^{\frac{1}{2}}$ as often considered, which applies to uniformly bright stars. In a rotating star with a variable local $T_{\text {eff }}$ in latitude, more care is needed. Due to the von Zeipel theorem, the critical velocity is equal to $v_{\text {crit }}=\left(\frac{G M}{R_{\mathrm{eb}}}\right)^{\frac{1}{2}}$ as long as $\Gamma \leq 0.639$, while above it, the critical velocity progressively decreases to zero (Maeder \& Meynet 2000).

In a rotating star, the mass flux is not constant over the stellar surface. At a colatitude $\vartheta$ the mass flux, i.e. the amount of mass loss $\Delta \dot{M}$ by surface element $\Delta \sigma$ is (Maeder \& Meynet 2000)

$$
\begin{array}{r}
\frac{\Delta \dot{M}(\vartheta)}{\Delta \sigma} \propto A\left[\frac{L(P)}{4 \pi G M_{\star}(P)}\right]^{\frac{1}{\alpha}} \frac{g_{\mathrm{eff}}\left[1+\zeta(\vartheta]^{\frac{1}{\alpha}}\right.}{\left[1-\Gamma_{\Omega}(\vartheta)\right]^{\frac{1}{\alpha}-1}} \\
\text { with } A=(k \alpha)^{\frac{1}{\alpha}}\left(\frac{1-\alpha}{\alpha}\right)^{\frac{1-\alpha}{\alpha}},
\end{array}
$$

$L(P)$ and $M_{\star}(P)$ are the luminosity and effective mass inside an equipotential (cf. Maeder \& Zahn 1998), here the surface equipotential. One has

$M_{\star}(P)=M\left(1-\frac{\Omega^{2}}{2 \pi G \rho_{\mathrm{m}}}\right)$.

The effective gravity $\boldsymbol{g}_{\text {eff }}$ includes the gravitational and centrifugal acceleration, while the total gravity $\boldsymbol{g}_{\text {tot }}=$ $\boldsymbol{g}_{\text {eff }}+\boldsymbol{g}_{\text {rad }}$ also accounts for the radiative acceleration. If we neglect the deviations from the von Zeipel theorem due to differential rotation, the term $\zeta(\vartheta)$ in Eq. (6) is equal to zero.
In a rotating star, the terminal velocity $v_{\infty}(\vartheta)$ depends on the colatitude $\vartheta$, it behaves like (Lamers \& Cassinelli 1999)

$$
\begin{aligned}
v_{\infty}(\vartheta) & \propto\left[g_{\mathrm{tot}}(\vartheta) R(\vartheta)\right]^{\frac{1}{2}} \\
& =\left[g_{\mathrm{eff}}(\vartheta)\left(1-\Gamma_{\Omega}\right) R(\vartheta)\right]^{\frac{1}{2}} .
\end{aligned}
$$

For $g_{\text {eff }}(\vartheta)$, we shall take below the radial component. Thus, we shall omit a factor $\cos \epsilon$ (cf. Maeder 1999), where $\epsilon$ is a small angle between the radial vector and the effective gravity. At most, $\cos \epsilon$ deviates from unity by $3 \%$. We get for the local mass flux-velocity-luminosity relation

$$
\frac{\Delta \dot{M}(\vartheta) v_{\infty}(\vartheta)}{\Delta \sigma} \propto\left[\frac{L(P)}{4 \pi G M_{\star}(P)}\right]^{\frac{1}{\alpha}} \frac{g_{\text {eff }}^{\frac{3}{2}}(\vartheta) R^{\frac{1}{2}}(\vartheta)}{\left[1-\Gamma_{\Omega}(\vartheta)\right]^{\frac{1}{\alpha}-\frac{3}{2}}}
$$

The modulus of the effective gravity in the radial direction is

$g_{\mathrm{eff}}(\vartheta)=\frac{G M}{R^{2}(\vartheta)}\left[1-w^{2}(\vartheta)\right]$

with $w^{2}(\vartheta)=\frac{\Omega^{2} R^{3}(\vartheta)}{G M} \sin ^{2} \vartheta$. The local WLR becomes

$$
\begin{aligned}
\dot{M}(\vartheta) v_{\infty}(\vartheta) & \propto \frac{L^{\frac{1}{\alpha}}(P)}{R^{\frac{1}{2}}(\vartheta)} \\
& \frac{\left[M\left(1-\Gamma_{\Omega}\right)\right]^{\frac{3}{2}-\frac{1}{\alpha}}\left[1-w^{2}(\vartheta)\right]^{\frac{3}{2}}}{\left(1-\frac{\Omega^{2}}{2 \pi G \rho_{\mathrm{m}}}\right)^{\frac{1}{\alpha}}} .
\end{aligned}
$$

Expressing $1-\Gamma_{\Omega}$, we finally get for the local WLR

$$
\begin{aligned}
& \dot{M}(\vartheta) v_{\infty}(\vartheta) \propto \\
& \frac{L^{\frac{1}{\alpha}}(P)}{R^{\frac{1}{2}}(\vartheta)}\left[M\left(1-\frac{\Omega^{2}}{2 \pi G \rho_{\mathrm{m}}}-\Gamma\right)\right]^{\frac{3}{2}-\frac{1}{\alpha}} f_{\omega}(\vartheta)
\end{aligned}
$$

with $f_{\omega}(\vartheta)$ given by

$$
f_{\omega}(\vartheta)=\frac{\left(1-w^{2}(\vartheta)\right)^{\frac{3}{2}}}{\left(1-\frac{\Omega^{2}}{2 \pi G \rho_{\mathrm{m}}}\right)^{\frac{3}{2}}}
$$

\subsection{Average orientation}

The previous expression gives the local WLR for a surface element of the star at a given colatitude $\vartheta$. We have to examine what is the average WLR and also how it depends on the orientation angle $i$ between the rotation axis and the line of sight. Let us firstly examine Eq. (13) for an average orientation. In the case of a random distribution of the orientation angles $i$, the average angle $\langle i\rangle$ is equal to 57.3 degrees. Let us consider for simplicity an average orientation corresponding to the vertical direction on a surface element at the zero of the Legendre Polynomial $P_{2}(\cos (\vartheta))=0$, i.e. for $\sin ^{2}(\vartheta)=2 / 3$, which corresponds to a colatitude of 54.7 degrees. This colatitude also corresponds to that of the average stellar radius in models with rotation (Meynet \& Maeder 1997). Let us call $R_{\mathrm{o}}$ this average stellar radius at the considered rotation, $R_{\mathrm{o}}$ is as a 
matter of fact the best estimate of the average radius of a rotating star. We have at the numerator of Eq. (13)

$$
\begin{aligned}
& 1-w^{2}=1-\frac{2}{3} \frac{\Omega^{2} R_{\mathrm{o}}^{3}}{G M} \\
& =1-\frac{4}{9} \frac{\Omega^{2} R_{\mathrm{pb}}^{2}}{v_{\mathrm{crit}}^{2}}\left(\frac{R_{\mathrm{o}}}{R_{\mathrm{pb}}}\right)^{3}=1-\frac{4}{9}\left(\frac{v}{v_{\text {crit }}}\right)^{2} \varphi(\omega),
\end{aligned}
$$

where $\varphi(\omega)$ is given by

$\varphi(\omega)=\left(\frac{R_{\mathrm{pb}}}{R_{\mathrm{e}}}\right)^{2}\left(\frac{R_{\mathrm{o}}}{R_{\mathrm{pb}}}\right)^{3}$.

$R_{\mathrm{pb}}$ is the polar radius at break-up, $R_{\mathrm{e}}$ is the equatorial radius at the considered rotational velocity $v=\Omega R_{\mathrm{e}}$. The ratio $\varphi(\omega)$ is derived from the Roche model; it is always slightly smaller than 1.0. For the denominator of Eq. (13), we have (Maeder \& Meynet 2000)

$$
\frac{\Omega^{2}}{2 \pi G \rho_{\mathrm{m}}}=\frac{4}{9} \frac{v^{2}}{v_{\mathrm{crit}}^{2}} V^{\prime}(\omega) \frac{R_{\mathrm{pb}}^{2}}{R_{\mathrm{e}}^{2}} .
$$

The volume $V^{\prime}(\omega)$ is the ratio of the actual volume of the rotating star to the spherical volume of radius $R_{\mathrm{pb}}$. The relation between $\frac{\Omega^{2}}{2 \pi G \rho_{\mathrm{m}}}$ and the ratio $\left(\frac{v}{v_{\text {crit }}}\right)^{2}$ is almost linear (Maeder \& Meynet 2000) and to a first order one has $\frac{\Omega^{2}}{2 \pi G \rho_{\mathrm{m}}}=\frac{4}{9}\left(\frac{v}{v_{\text {crit }}}\right)^{2}$. For the average orientation defined above, the wind momentum-luminosity relation is thus

$\dot{M} v_{\infty} \propto \frac{L^{\frac{1}{\alpha}}(P)}{R_{\mathrm{o}}^{\frac{1}{2}}}\left[M\left(1-\frac{\Omega^{2}}{2 \pi G \rho_{\mathrm{m}}}-\Gamma\right)\right]^{\frac{3}{2}-\frac{1}{\alpha}} f_{\omega}$.

For OB stars, the force multiplier $\alpha$ is very close to $2 / 3$ and one is left with

$\dot{M} v_{\infty} \propto \frac{L^{\frac{1}{\alpha}}(P)}{R_{\mathrm{O}}^{\frac{1}{2}}} f_{\omega}$.

Equations (14) and (16) enable us to calculate the fraction $f_{\omega}$ in Eq. (17). The variations of $f_{\omega}$ as a function of $\frac{v}{v_{\text {crit }}}$ are shown in Fig. 1. We see that, up to a fraction 0.6 of the critical velocity, $f_{\omega}$ does not deviate from 1 by more than $1 \%$, up to a fraction 0.8 of the critical velocity the maximum deviation is $4 \%$, and for a fraction 0.9 it is $9 \%$. Since in Eq. (18), the luminosity appears with an exponent $1 / \alpha$, the effects on the luminosity determinations are even smaller. As an example, for a fraction 0.8 of the critical velocity, the luminosities which would be derived from the usual Eq. (1) would be too large by $2.6 \%$, and by $5.9 \%$ for a fraction 0.9 of the critical velocity. This means that for given $\dot{M} v_{\infty}$ and radii, the determinations of the luminosity is not very much modified by rotation, even if it is very fast. The function $f_{\omega}$ behaves like $f_{\omega}=1.17\left(\frac{v}{v_{\text {crit }}}\right)^{6}$ with deviations smaller than 0.002 up to $\frac{v}{v_{\text {crit }}}=0.97$.

It is remarquable that, while all quantities in Eq. (18) are substantially influenced by rotation (in particular the mass loss rates), the overall relation between the actual mass loss rates, terminal velocities, luminosities and radii is the same for OB stars. We conclude that, because of compensation effects, the effects of rotation are remarkably absent in the average wind-momentum relation for OB stars.

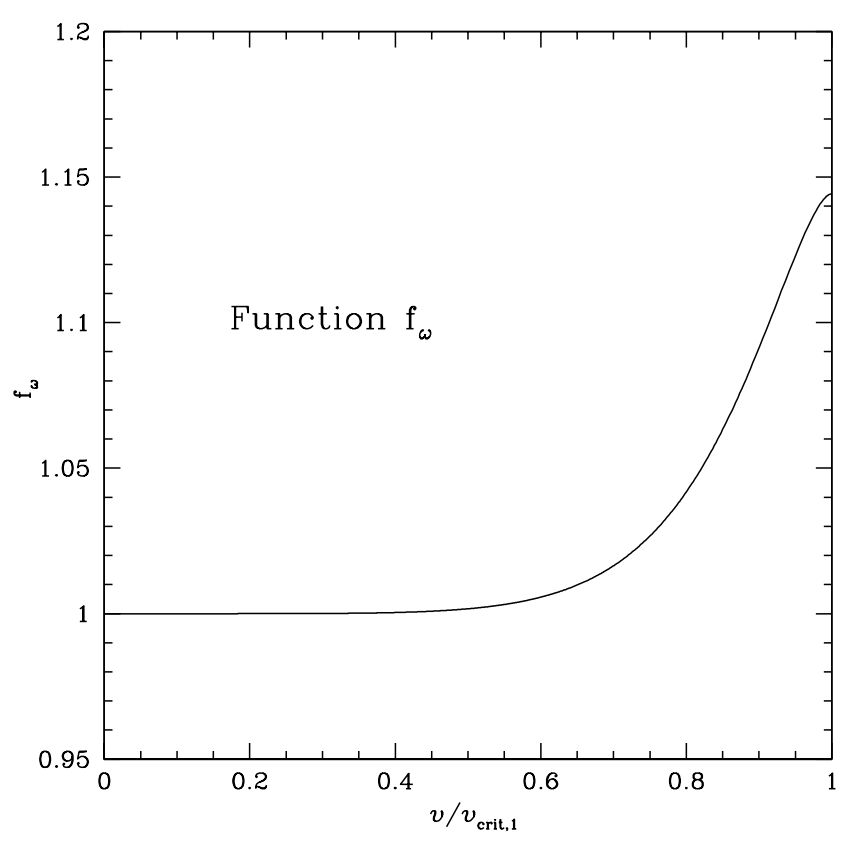

Fig. 1. The function $f_{\omega}$ which expresses the deviations from the usual WLR is shown in terms of the fraction of the critical velocity, (cf. Eq. (18)).

\subsection{Orientation effects}

We need also to estimate the size of the effects of orientation, which could produce some scatter around the mean WLR given above. Let us consider the extreme cases of regions at the pole and at the equator. Firstly, we notice that at the pole, the values of $f_{\omega}(\vartheta)$ become bigger than 1.0 for growing rotations. For example, for values of the fraction of the break-up angular velocity $\omega=0.5,0.8$, one respectively has $f_{\omega}$ (pole) $=1.086$ and 1.305 . On the contrary, at the equator, the values $f_{\omega}(\vartheta)$ becomes smaller than 1.0 for growing rotations. For example, for $\omega=0.5,0.8$, one respectively has $f_{\omega}$ (equator) $=0.952$ and 0.796 . This means that locally, the deviations from the WLR due to the factor $f_{\omega}(\vartheta)$ are not negligible.

Now, we must ensure that the observable effects are integrated over the visible part of the stellar surface; this produces an averaging of regions with different values of $f_{\omega}(\vartheta)$. Let us do it for the extreme cases of pole-on and of equator-on observers. For an observer who is seeing poleon a rotating star with $\omega=0.5,0.8$ and 0.9 , the apparent surface weighted values of $f_{\omega}$ (pole-on) are $1.021,1.068$ and 1.103 respectively. This shows that the luminosities derived by the application of the usual Eq. (1) are too large by $1.4,4.5$ and $6.8 \%$ respectively.

For an observer who is seeing equator-on a rotating star with $\omega=0.5,0.8$ and 0.9 , the apparent surface weighted values of $f_{\omega}$ (equator-on) are $0.987,0.934$ and 0.878 respectively. These last values imply that the luminosities which would be derived by the application of the usual Eq. (1) would be too small by $0.9,4.5$ and $8.3 \%$ respectively. This shows that, even for very fast rotations, 
the deviations from the average WLR relation, due to orientation effects, are relatively small.

Finally, we may also consider a weighting according to the local flux emitted. The results are very much of the same order. As an example, for pole-on observations, the deviations for $\omega=0.9$ are smaller than $2.5 \%$. For an equator-on observer, the luminosities which would derived by the application of the usual Eq. (1) would be too small by $1.7,7.5$ and $13.7 \%$ for $\omega=0.5,0.8$ and 0.9 respectively. Thus, whatever the weighting function we consider, we conclude that the errors on the luminosity determinations due to rotation effects are very limited.

\section{Conclusions}

The effects of stellar rotation on the WLR are generally very small. For an average orientation angle, their amplitudes remain negligible for most rotation velocities. Different orientations angles of the rotation axis with respect to the line of sight can produce some limited scatter on the luminosity determinations. We estimate the scatter in the luminosities introduced by orientation effects to be at most of the order of $10 \%$.

Here, we have made an analytical approach to the problem. This does not preclude more complete numerical simulations, as made by Petrenz \& Puls (2000), who performed 2-D non-LTE models of rotating early-type stars.

Acknowledgements. I express my best thanks to Dr. Georges Meynet for most fruitful discussions.

\section{References}

Keller, S. C. 1999, Thesis, Mount Stromlo Observatory

Kudritzki, R. P. 2000, in Unsolved Problems in Stellar Evolution, ed. M. Livio, STScI Ser., 12, 202

Kudritzki, R. P., Pauldrach, A. W., Puls, J., \& Abbott, D. C. 1989, A\&A, 219, 205

Kudritzki, R. P., Puls, J., Lennon, D. J., et al. 1999, A\&A, 350,970

Kudritzki, R. P., \& Puls, J. 2000, ARA\&A, 38, 613

Lamers, H. J. G. L. M., \& Cassinelli, J. P. 1999, Introduction to Stellar Winds (Cambridge Univ. Press), 328

Maeder, A. 1999, A\&A, 347, 185

Maeder, A., Grebel, E., \& Mermilliod, J. C. 1999, A\&A, 346, 459

Maeder, A., \& Meynet, G. 2000, A\&A, 361, 159

Maeder, A., \& Zahn, J. P. 1998, A\&A, 334, 1000

Meynet, G., \& Maeder, A. 1997, A\&A, 321, 465

Petrenz, P., \& Puls, J. 2000, A\&A, 358, 956

Puls, J., Kudritzki, R. P., Herrero, A., et al. 1996, A\&A, 305, 171 